\title{
A general existence theorem of zero points
}

Citation for published version (APA):

Herings, P. J. J., Koshevoy, G., Talman, A. J. J., \& Yang, Z. (2002). A general existence theorem of zero points. METEOR, Maastricht University School of Business and Economics. METEOR Research Memorandum No. 049 https://doi.org/10.26481/umamet.2002049

Document status and date:

Published: 01/01/2002

DOI:

10.26481/umamet.2002049

Document Version:

Publisher's PDF, also known as Version of record

\section{Please check the document version of this publication:}

- A submitted manuscript is the version of the article upon submission and before peer-review. There can be important differences between the submitted version and the official published version of record.

People interested in the research are advised to contact the author for the final version of the publication, or visit the DOI to the publisher's website.

- The final author version and the galley proof are versions of the publication after peer review.

- The final published version features the final layout of the paper including the volume, issue and page numbers.

Link to publication

\footnotetext{
General rights rights.

- You may freely distribute the URL identifying the publication in the public portal. please follow below link for the End User Agreement:

www.umlib.nl/taverne-license

Take down policy

If you believe that this document breaches copyright please contact us at:

repository@maastrichtuniversity.nl

providing details and we will investigate your claim.
}

Copyright and moral rights for the publications made accessible in the public portal are retained by the authors and/or other copyright owners and it is a condition of accessing publications that users recognise and abide by the legal requirements associated with these

- Users may download and print one copy of any publication from the public portal for the purpose of private study or research.

- You may not further distribute the material or use it for any profit-making activity or commercial gain

If the publication is distributed under the terms of Article $25 \mathrm{fa}$ of the Dutch Copyright Act, indicated by the "Taverne" license above, 


\title{
A General Existence Theorem of Zero Points ${ }^{1}$
}

\author{
P. Jean-Jacques Herings ${ }^{2}$ and Gleb A. Koshevoy ${ }^{3}$ \\ Dolf Talman ${ }^{4}$ and Zaifu Yang ${ }^{5}$
}

November 27, 2002

\footnotetext{
${ }^{1}$ This research is part of the Research Program "Competition and Cooperation." It has been carried out while the second author was visiting CentER, Tilburg University, on an NWO-grant. The fourth author is also supported by the Alexander von Humboldt Foundation.

${ }^{2}$ P.J.J. Herings, Department of Economics, Universiteit Maastricht, P.O. Box 616, 6200 MD Maastricht, The Netherlands. E-mail: P.Herings@algec.unimaas.nl

${ }^{3}$ G.A. Koshevoy, Central Institute of Mathematics and Economics, Russian Academy of Sciences, 117418 Moscow, Russia. E-mail: koshevoy@cemi.rssi.ru

${ }^{4}$ A.J.J. Talman, Department of Econometrics \& Operations Research and CentER, Tilburg University, P.O. Box 90153, 5000 LE Tilburg, The Netherlands. E-mail: talman@uvt.nl

${ }^{5}$ Z. Yang, Institute of Mathematical Economics, University of Bielefeld, 33615 Bielefeld, Germany. E-mail: zyang@wiwi.uni-bielefeld.de; and Faculty of Business Administration, Yokohama National University, Yokohama 240-8501, Japan. E-mail: zyang@business.ynu.ac.jp
} 


\begin{abstract}
Let $X$ be a non-empty, compact, convex set in $\mathbb{R}^{n}$ and $\phi$ an upper semi-continuous mapping from $X$ to the collection of non-empty, compact, convex subsets of $\mathbb{R}^{n}$. It is well known that such a mapping has a stationary point on $X$, i.e. there exists a point in $X$ satisfying that its image under $\phi$ has a non-empty intersection with the normal cone of $X$ at the point. In case for every point in $X$ it holds that the intersection of the image under $\phi$ with the normal cone of $X$ at the point is either empty or contains the origin $0^{n}$, then $\phi$ must have a zero point on $X$, i.e. there exists a point in $X$ satisfying that $0^{n}$ lies in the image of the point. Another well-known condition for the existence of a zero point follows from Ky Fan's coincidence theorem, which says that if for every point the intersection of the image with the tangent cone of $X$ at the point is non-empty, the mapping must have a zero point. In this paper we extend all these existence results by giving a general zero point existence theorem, of which the two results are obtained as special cases. We also discuss what kind of solutions may exist when no further conditions are stated on the mapping $\phi$. Finally, we show how our results can be used to establish several new intersection results on a compact, convex set.
\end{abstract}

Keywords: stationary point, zero point, fixed point, normal cone, tangent cone, intersection point. 


\section{Introduction}

Whenever a mathematical model of some phenomenon is constructed, for instance, in engineering or in economics, the first question to ask is whether a solution to the model exists. A very powerful tool that is often used to this end in case the model is a system of nonlinear functions is Brouwer's fixed point theorem; see Brouwer (1912). When the model is not a system of equations but a system of correspondences, often Kakutani's fixed point theorem (1941) is invoked. Sometimes, models allow for a continuum of solutions, and appropriate generalizations of Brouwer's and Kakutani's fixed point theorems as provided by Browder (1960) and Herings, Talman, and Yang (1996, 2001) apply. An alternative to fixed point theorems consists of using intersection theorems, with the lemma of Knaster, Kuratowski and Mazurkiewicz (1929) on the unit simplex perhaps the most prominent example.

The existence of a solution to a nonlinear system of functions or correspondences is equivalent to the existence of a zero point of a function or a correspondence. A zero point is a point in the domain satisfying that the origin lies in its image. In this paper we will present a new general condition for the existence of a zero point.

Let $X$ be a nonempty convex and compact set in $\mathbb{R}^{n}$ and let $\phi$ be a compact-valued, convex-valued upper-semicontinuous mapping from $X$ to $\mathbb{R}^{n}$. By Eaves (1971) it has been shown that with respect to any such correspondence a solution exists to the variational inequality problem, i.e. there exists a stationary point. Such a point $x$ in $X$ satisfies that its image $\phi(x)$ has a nonempty intersection with the normal cone $N(X, x)$ to $X$ at $x$. From this it immediately follows that if at every point of $X$ the intersection of the image and the normal cone is either empty or contains the origin, then $\phi$ has a zero point in $X$. Fan (1972) proved a coincidence result, stating a weakly seperating condition under which there is a point $x$ in $X$ such that $\phi(x)$ has a nonempty intersection with the image at $x$ of some other correspondence $\psi$ on $X$. This condition also makes use of the normal cone at any point in $X$. When $\psi$ maps every point of $X$ to the origin and the seperating condition is satisfied, a zero point of $\phi$ exists.

In this paper we present a unifying theorem on the existence of zero points. The theorem puts two conditions on $\phi(x)$ at every $x$ in $X$. Both conditions are related to the normal cone. More precisely, the conditions put restrictions on the set $A \phi(x) \cap \pi(v)$, where $A$ is a nonsingular $n \times n$-matrix, $v$ is any normalized element of the normal cone at $x$, and $\pi$ is an upper hemi-continuous correspondence defined on the unit ball. The new theorem contains the two existence results for zero points mentioned above as special cases. The stationary point condition is obtained when $A$ is the identity matrix and $\pi(v)$ is equal to $\mathbb{R}^{n}$ for every $v$, while the coincidence point condition is obtained by also taking $A$ equal to the identity matrix and $\pi(v)$ equal to the set $\left\{y \in \mathbb{R}^{n} \mid y^{\top} v \leq 0\right\}$. Other choices for the 
matrix $A$ and the correspondence $\pi$ lead to different and new zero point existence theorems. Further we show how Kakutani's fixed point theorem as well as other fixed point theorems on unbounded domains, as presented in Merrill (1971) and Eaves (1972), can be obtained as special cases of our main result. We also generalize the notion of stationary point in case for at least one point in $X$ the two conditions are satisfied for no correspondence $\pi$. These results are exemplified in Sections 2 and 3.

Section 4 treats the special case of the zero point problem when $X$ is a polytope. The special structure of the polytope is exploited to obtain a sharp result on the existence of a zero point. Section 5 shows how a more general intersection theorem can be derived from our main theorem on the existence of zero points. This general intersection theorem contains several well known intersection theorems like the ones of Knaster, Kuratowski, and Mazurkiewicz (1929), Scarf (1967), Shapley (1973), and Ichiishi (1988) as special cases.

\section{The Zero Point Problem}

Consider an arbitrary non-empty, convex, compact set $X$ in the $n$-dimensional Euclidean space $\mathbb{R}^{n}$. For $x \in X$, the set $N(X, x)=\left\{y \in \mathbb{R}^{n} \mid\left(x-x^{\prime}\right)^{\top} y \geq 0\right.$ for all $\left.x^{\prime} \in X\right\}$ denotes the normal cone of the set $X$ at the point $x$. Since $X$ is compact and convex, $N(X, \cdot)$ is an upper semi-continuous, convex-valued and closed-valued mapping.

Let $\phi$ be a point-to-set mapping or correspondence from $X$ to the collection of nonempty subsets of $\mathbb{R}^{n}$. We assume that $\phi$ is an upper semi-continuous and bounded mapping and that for every $x$ in $X$ the set $\phi(x)$ is a compact and convex subset of $\mathbb{R}^{n}$. We are interested in conditions on the mapping $\phi$, under which $\phi$ has a zero point, being a point $x^{*}$ in $X$ satisfying $0^{n} \in \phi\left(x^{*}\right)$, where $0^{n}$ is the $n$-vector of zeroes. Without any conditions on $\phi$ a zero point may not exist. However, as has been shown in Eaves (1971), a stationary point of $\phi$ on $X$ always exists.

Definition 2.1 A point $x^{*} \in X$ is a stationary point of $\phi$ if there exists $y^{*} \in \phi\left(x^{*}\right)$ such that $\left(x^{*}-x\right)^{\top} y^{*} \geq 0$ for all $x \in X$, i.e. $\phi\left(x^{*}\right) \cap N\left(X, x^{*}\right) \neq \emptyset$.

From Eaves' result it immediately follows that if all stationary points of $\phi$ are zero points of $\phi$, then $\phi$ has at least one zero point.

Theorem 2.2 If for every $x \in X$ it holds that $\phi(x) \cap N(X, x)$ is either empty or contains $0^{n}$, then there exists a zero point of $\phi$.

The condition in the theorem says that a zero point of $\phi$ exists if at any $x \in X$ no nonzero element of the image $\phi(x)$ lies in the normal cone of $X$ at $x$ unless the image contains $0^{n}$. Although this condition is rather weak it has to hold for all elements in every image set. 
Another existence result for zero points can be obtained from the coincidence theorem of Fan (1972).

Definition 2.3 Let $\phi$ and $\psi$ be to correspondences from $X$ to $\mathbb{R}^{n}$. A point $x^{*} \in X$ is a coincidence point of $\phi$ and $\psi$ if $\phi\left(x^{*}\right) \cap \psi\left(x^{*}\right) \neq \emptyset$.

By Fan (1972) it has been proved that if $\psi$ is also an upper semi-continuous, bounded, convex-valued and compact-valued correspondence from $X$ to $\mathbb{R}^{n}$ and for every $x \in X$ and every $v \in N(X, x)$ there exists $y \in \phi(x)$ and $z \in \psi(x)$ such that $v^{\top} y \leq v^{\top} z$, then $\phi$ and $\psi$ have a coincidence point. By taking $\psi(x)$ equal to $\left\{0^{n}\right\}$ for all $x$ in $X$, we obtain the following zero point result, which is an equivalent form of Fan's coincidence theorem.

Theorem 2.4 If for every $x \in X$ and every $v \in N(X, x)$ there exists $y \in \phi(x)$ such that $v^{\top} y \leq 0$, then $\phi$ has a zero point.

The condition in this theorem says that for every $x$ in $X$ the set $\phi(x)$ should have a nonempty intersection with any halfspace that is the polar (or dual) cone of an element of the normal cone of $X$ at $x$. The fact that two rather different conditions lead to the same existence result suggests a more general zero point existence result. In the next section we give a zero point existence theorem that contains both theorems above and several other known existence results as special cases.

\section{The Existence Results}

In this section we give a unifying zero point existence result on a compact, convex set. Both Theorems 2.2 and 2.4 as well as Kakutani's fixed point theorem and other fixed and zero point theorems are special cases of this theorem. As in Section 2 we assume that the set $X$ is a non-empty compact and convex subset of $\mathbb{R}^{n}$ and that $\phi$ is an upper semicontinuous, bounded, compact-valued and convex-valued correspondence from $X$ to $\mathbb{R}^{n}$. Let $B^{n}$ denote the $n$-dimensional unit ball.

Theorem 3.1 Suppose that there exists a non-singular $n \times n$ matrix $A$ and an upper semi-continuous, convex-valued, closed-valued mapping $\pi: B^{n} \rightarrow \mathbb{R}^{n}$ such that for every $x \in X$ and every $v \in N(X, x) \cap B^{n}$ the following two properties hold:

1. The set $A \phi(x) \cap \pi(v) \cap\{y \mid y=\mu v, \mu \geq 0\}$ is either empty or contains $0^{n}$;

2. The set $A \phi(x) \cap \pi(v) \neq \emptyset$.

Then there exists a zero point of $\phi$ in $X$. 
Proof: Let the set $Q$ be defined by

$$
Q=\left\{q \in \mathbb{R}^{n} \mid\|q-x\|_{2} \leq 1 \text { for some } x \in X\right\}
$$

Since $X$ is compact, $Q$ is a compact set. For $q \in Q$, let $p(q)$ be the orthogonal projection of $q$ on $X$. Since $X$ is a non-empty, compact, convex set, $p$ is a continuous function from $Q$ to $X$. For every $q \in Q$ it holds that

$$
\|q-p(q)\|_{2} \leq 1
$$

To prove convexity of $Q$, take any $q^{1}, q^{2} \in Q$ and $0 \leq \lambda \leq 1$, and let

$$
q(\lambda)=\lambda q^{1}+(1-\lambda) q^{2}
$$

and

$$
p(\lambda)=\lambda p\left(q^{1}\right)+(1-\lambda) p\left(q^{2}\right) .
$$

Since $X$ is convex, we have that $p(\lambda) \in X$. Moreover,

$$
\|q(\lambda)-p(\lambda)\|_{2} \leq \lambda\left\|q^{1}-p\left(q^{1}\right)\right\|_{2}+(1-\lambda)\left\|q^{2}-p\left(q^{2}\right)\right\|_{2} \leq 1
$$

Therefore, $q(\lambda) \in Q$, i.e. $Q$ is a convex set. Hence, $Q$ is a full-dimensional compact, convex set in $\mathbb{R}^{n}$. For $q \in Q$, let $v(q)=q-p(q)$. By construction, $v(q) \in B^{n}$ for each $q \in Q$, $\|v(q)\|_{2}=1$ if and only if $q \in \operatorname{bd}(Q)$, and $v(q)=0^{n}$ if and only if $q \in X$. Since $Q$ is full-dimensional, for $q \in \operatorname{int}(Q)$ it holds that $N(Q, q)=\left\{0^{n}\right\}$. Now we will show that the normal cone $N(Q, q)$ of $Q$ at any point $q$ on the boundary of $Q$ is a ray. Since $Q$ is convex and compact, $N(Q, q)$ is nonempty for every $q \in Q$. Take any point $q \in \operatorname{bd}(Q)$ and consider the ball $B(p(q), 1)$ with radius one centered at $p(q)$. Clearly, $B(p(q), 1)$ is contained by $Q$ and $q$ also lies on the boundary of $B(p(q), 1)$. It follows that $N(Q, q)$ is a subset of $N(B(p(q), 1), q)$. Since the boundary of $B(p(q), 1)$ is smooth, $N(B(p(q), 1), q)$ is a ray. Consequently, $N(Q, q)$ must be a ray as well and in fact is equal to $N(B(p(q), 1), q)$. More precisely, for $q \in \operatorname{bd}(Q)$ we have

$$
N(Q, q)=\left\{y \in \mathbb{R}^{n} \mid y=\mu v(q), \mu \geq 0\right\} .
$$

Since $p$ is the orthogonal projection on $X$, for every $q \in Q$ it holds that $N(Q, q) \subset$ $N(X, p(q))$. Now consider the mapping $\psi: Q \rightarrow \mathbb{R}^{n}$ defined by

$$
\psi(q)=A \phi(p(q)) \cap \pi(q-p(q))
$$

From Condition 2 it follows that for every $q \in Q$ the set $\psi(q)$ is non-empty. Since $A$ is a regular matrix, $p$ is a continuous function and both $\phi$ and $\pi$ are upper semi-continuous mappings, $\psi$ is an upper semi-continuous mapping from the full-dimensional, compact, 
convex set $Q$ to $\mathbb{R}^{n}$. Moreover, being the intersection of a convex and compact set and a convex and closed set, $\psi(q)$ is convex and compact for any $q \in Q$. From Eaves (1971) it now follows that $\psi$ has a stationary point on $Q$, i.e., there exists a point $q^{*} \in Q$ such that

$$
\psi\left(q^{*}\right) \cap N\left(Q, q^{*}\right) \neq \emptyset
$$

Take any $f^{*}$ in this intersection. Since $f^{*} \in N\left(Q, q^{*}\right)$ it holds that $f^{*}=\mu^{*} v\left(q^{*}\right) \in$ $N\left(X, p\left(q^{*}\right)\right)$ for some $\mu^{*} \geq 0$. Hence,

$$
f^{*} \in\left\{\mu v\left(q^{*}\right) \mid \mu \geq 0\right\} \cap A \phi\left(p\left(q^{*}\right)\right) \cap \pi\left(v\left(q^{*}\right)\right),
$$

with $v\left(q^{*}\right) \in N\left(X, p\left(q^{*}\right)\right) \cap B^{n}$. Since we showed that the intersection of these three sets is non-empty, Condition 1 implies that this intersection contains $0^{n}$, from which we conclude that $p\left(q^{*}\right)$ is a zero point of $A \phi$ on $X$. Since $A$ is a nonsingular matrix, $p\left(q^{*}\right)$ is a zero point of $\phi$.

The theorem says that the mapping $\phi$ has a zero point on $X$, if there exists a regular matrix $A$ and an upper semi-continuous, convex-valued and closed-valued mapping on the unit ball $B^{n}$ satisfying that for every element $v$ of the normal cone of $X$ at any $x$ with length at most one the image of $A \phi$ at $x$ and the image of $\pi$ at $v$ intersect, but this intersection has no points in common with the ray determined by the vector $v$ unless the origin is in the intersection. In case $\phi$ is a continuous function $f$ from $X$ to $\mathbb{R}^{n}$, Conditions 1 and 2 reduce to for every $x \in X$ and $v \in N(X, x) \cap B^{n}$ it holds that $A f(x) \in \pi(v)$ and Af $(x) \notin(\pi(v) \cap\{y \mid y=\mu v, \mu \geq 0\}) \backslash\left\{0^{n}\right\}$.

Instead of taking a mapping on the whole unit ball, we may restrict ourselves to a mapping $\pi$ on the sphere $\operatorname{bd}\left(B^{n}\right)$. The proof is then the same, by extending the mapping $\pi$ to the whole unit ball as follows: $\pi\left(0^{n}\right)$ contains every $\pi(v), v \in B^{n}$, and $\pi(v)=\pi\left(v /\|v\|_{2}\right)$ for $v \in \operatorname{int}\left(B^{n}\right) \backslash\left\{0^{n}\right\}$. The matrix $A$ translates the images $\phi(x)$ in a linear way, so that $A \phi(x)$ has the same properties as $\phi(x)$ has. Due to the regularity of $A$ a point $x^{*}$ is a zero point of $\phi$ if and only if $x^{*}$ is a zero point of $A \phi$.

The use of the matrix $A$ expands the cases to which our result applies. For example, consider the function $f: B^{n} \rightarrow \mathbb{R}^{n}$ defined by $f(x)=x$. Then there is no mapping $\pi$ that satisfies both conditions 1 and 2, although $f\left(0^{n}\right)=0^{n}$. However, when we take $A=-I$, where $I$ is the $n \times n$ identity matrix, conditions 1 and 2 are satisfied if we take for example $\pi(v)=\mathbb{R}^{n}$ for all $v \in B^{n}$.

In the following we will show that several known existence results are special cases of Theorem 3.1.

Example 3.2 When $\pi(v)=\mathbb{R}^{n}$ for every $v \in B^{n}$, then Condition 2 of Theorem 3.1 is always satisfied and Condition 1 reduces to $A \phi(x) \cap N(X, x)$ is empty or contains $0^{n}$, 
for every $x \in X$. For $A=I$ this is precisely the condition of Theorem 2.2. However, the result holds for any regular matrix $A$, e.g. a zero point also exists when for every $x \in X$ it holds that $-\phi(x) \cap N(X, x)$ is either empty or contains $0^{n}$.

Example 3.3 When $\pi(v)=\left\{y \in \mathbb{R}^{n} \mid y^{\top} v \leq 0\right\}$, for every $v \in B^{n}$, then

$$
\pi(v) \cap\left\{y \in \mathbb{R}^{n} \mid y=\mu v, \mu \geq 0\right\}=\left\{0^{n}\right\},
$$

for any $v \in B^{n}$, and so Condition 1 of Theorem 3.1 is always satisfied, while when $A=I$ Condition 2 becomes precisely the condition of Theorem 2.4. Also now, the result holds for any regular matrix $A$.

Thus, both Theorem 2.2 and Theorem 2.4 are special cases of Theorem 3.1. For $x \in X$, let the tangent cone of $X$ at $x$ be defined by

$$
T(X, x)=\left\{z \in \mathbb{R}^{n} \mid z^{\top} y \leq 0 \text {, for all } y \in N(X, x)\right\} .
$$

The next result says that $\phi$ has a zero point if for every $x$ in $X$ the set $\phi(x)$ has a non-empty intersection with $T(X, x)$.

Theorem 3.4 If for every $x \in X$ it holds that $\phi(x) \cap T(X, x) \neq \emptyset$, then $\phi$ has a zero point.

Proof: We show that the conditions of Theorem 3.1 are satisfied for $\pi(v)=\left\{y \in \mathbb{R}^{n} \mid\right.$ $\left.y^{\top} v \leq 0\right\}$ and $A=I$, and so $\phi$ has a zero point. Condition 1 of Theorem 3.1 is satisfied because $\pi(v) \cap\{y \mid y=\mu v, \mu \geq 0\}=\left\{0^{n}\right\}$, so $\phi(x) \cap \pi(v) \cap\{y \mid y=\mu v, \mu \geq 0\}$ is either empty or contains $\left\{0^{n}\right\}$. When $v \in N(X, x)$, it follows that $T(X, x) \subset \pi(v)$, so $\phi(x) \cap \pi(v) \neq \emptyset$ if $\phi(x) \cap T(X, x) \neq \emptyset$, and Condition 2 follows.

Obviously, $\phi$ has also a zero point on $X$ if there exists a regular matrix $A$ satisfying that $A \phi(x) \cap T(X, x) \neq \emptyset$, for every $x \in X$. The condition in Theorem 3.4 is very simple and in general easy to check. From Theorem 3.4 we immediately get Kakutani's fixed point theorem.

Example 3.5 Kakutani's fixed point theorem states that if $\phi$ is a correspondence from $X$ into itself it has at least one fixed point, i.e., there exists $x^{*} \in X$ satisfying $x^{*} \in \phi\left(x^{*}\right)$. Define the mapping $\psi$ from $X$ to $\mathbb{R}^{n}$ by $\psi(x)=\phi(x)-\{x\}$ for all $x \in X$. Since $X-\{x\} \subset T(X, x)$ and $\phi(x) \subset X$ for all $x \in X$, we have that $\psi(x) \subset T(X, x)$ and so $\psi(x) \cap T(X, x) \neq \emptyset$ for all $x \in X$. From Theorem 3.4 it follows that there exists $x^{*} \in X$ such that $0^{n} \in \psi\left(x^{*}\right)$. Clearly, $x^{*}$ is a fixed point of $\phi$.

The set $\pi(v)$ is not necessarily a half-space or the whole space as is illustrated in the next example. 
Example 3.6 Fix some strictly positive vector $m \in \mathbb{R}^{n}$. Let $\pi(v)$ be given by $\pi\left(0^{n}\right)=$ $\mathbb{R}^{n}$ and, for $v \in B^{n} \backslash\left\{0^{n}\right\}$, by

$$
\pi(v)=\left\{y \in \mathbb{R}^{n} \mid y_{i} \leq m_{i}\left(1-v_{i} / \max _{j}\left|v_{j}\right|\right) \text { if } v_{i}>0, y_{i} \geq m_{i}\left(-1-v_{i} / \max _{j}\left|v_{j}\right|\right) \text { if } v_{i}<0\right\} .
$$

Clearly, $\pi$ is an upper semi-continuous, convex-valued, and closed-valued correspondence on $B^{n}$. Moreover, for every $v \in B^{n}$ it holds that

$$
\pi(v) \cap\left\{y \in \mathbb{R}^{n} \mid y=\mu v, \mu \geq 0\right\}=\left\{0^{n}\right\},
$$

and so Condition 1 of Theorem 3.1 is always satisfied. If Condition 2 holds for this $\pi$, then there exists a zero point of $\phi$ on $X$.

The result in Example 3.6 was introduced in Herings, van der Laan and Talman (2001) to prove the existence of a continuum of quantity constrained equilibria in an exchange economy with prices restricted to an arbitrary convex, compact set. The next fixed point theorem is due to Eaves (1972) and is used to guarantee the convergence of simplicial homotopy algorithms.

Example 3.7 Let $X$ be full-dimensional and suppose there exists $c \in \operatorname{int}(X)$ such that for all $x \in \operatorname{bd}(X)$ it holds that $c \in \phi(x)$, then there exists a fixed point of $\phi$ in $X$. Define $\psi(x)=\phi(x)-\{x\}$ for all $x \in X$. For $x \in \operatorname{bd}(X)$ it holds that $c-x \in T(X, x) \cap \psi(x)$ and for $x \in \operatorname{int}(X)$ it holds that $T(X, x)=\mathbb{R}^{n}$. Hence, $T(X, x) \cap \psi(x) \neq \emptyset$ for every $x \in X$, i.e. the mapping $\psi$ satisfies the condition of Theorem 3.4. Therefore, $\psi$ has a zero point on $X$, which is a fixed point of $\phi$.

The following fixed point theorem is due to Merrill (1971) and has also applications in constrained and unconstrained optimization.

Example 3.8 Let $\psi$ be an upper semi-continuous mapping from $\mathbb{R}^{n}$ to the collection of compact, convex subsets of $\mathbb{R}^{n}$. Suppose there exists $w \in \mathbb{R}^{n}$ and $\mu>0$ satisfying for all $x \notin B(w, \mu)$ and $f \in \psi(x)$

$$
(f-x)^{\top}(w-x)>0,
$$

then $\psi$ has a fixed point in $B(w, \mu)$. Take $X=B(w, \mu)$ and $\pi(v)=\left\{y \in \mathbb{R}^{n} \mid y^{\top} v \leq 0\right\}$ for $v \in B^{n}$. For $x$ on the boundary of $B(w, \mu)$, it holds that $x-w \in N(X, x)$ and there is $f \in \psi(x)$ such that $(f-x)^{\top}(w-x) \geq 0$. For those $x$, Condition 2 of Theorem 3.1 holds for the mapping $\phi$ on $X$ defined by $\phi(x)=\psi(x)-\{x\}$. For $v$ in the interior of $B(w, \mu)$, Condition 2 is trivially satisfied. Also Condition 1 is satisfied for $\phi$, since for every $x \in \operatorname{bd}(X)$ it holds that $N(X, x)=\left\{y \in \mathbb{R}^{n} \mid y=\mu(x-w), \mu \geq 0\right\}$ and $(x-w)^{\top}(x-w)>0$. Hence, there exists $x^{*}$ in $X$ satisfying $0^{n} \in \phi\left(x^{*}\right)$, and therefore $x^{*} \in \psi\left(x^{*}\right)$. 
In Theorem 3.1 we have provided a sufficient condition for the existence of a zero point of a mapping on an arbitrary compact and convex set. In case the conditions of Theorem 3.1 are not satisfied, a zero point may not exist. In this case, it is possible to obtain a generalization of the notion of a stationary point, without losing existence of a stationary point under standard assumptions.

Definition 3.9 Let $\pi: B^{n} \rightarrow \mathbb{R}^{n}$ be a convex-valued, closed-valued, upper semicontinuous mapping and let $A$ be any nonsingular $n \times n$ matrix. A point $x^{*} \in X$ is a stationary point with respect to $\pi$ and $A$ of the mapping $\phi$ from $X$ to $\mathbb{R}^{n}$ if $0^{n} \in \phi\left(x^{*}\right)$ or $A \phi\left(x^{*}\right) \cap \pi(v) \cap\{y \mid y=\mu v, \mu \geq 0\} \neq \emptyset$ for some $v \in N\left(X, x^{*}\right)$ or $A \phi\left(x^{*}\right) \cap \pi(v)=\emptyset$ for some $v \in N\left(X, x^{*}\right)$.

Notice that when $\pi(v)=\mathbb{R}^{n}$ for all $v \in B^{n}$, and $A$ is the identity matrix, then the above definition is reduced to the usual definition of a stationary point; see Definition 2.1. As a consequence of Theorem 3.1, we have:

Theorem 3.10 Let $X$ be non-empty, compact, and convex, and let $\phi$ be upper semicontinuous, bounded, convex-valued, and compact-valued. Then, for every convex-valued, closed-valued, upper semi-continuous mapping $\pi: B^{n} \rightarrow \mathbb{R}^{n}$, and for every nonsingular $n \times n$ matrix $A, \phi$ has a stationary point with respect to $\pi$ and $A$.

\section{Zero Points on Polytopes}

In this section we consider the case that the compact, convex set $X$ is a polytope. Let a polytope $P$ be described in polyhedral form by

$$
P=\left\{x \in \mathbb{R}^{n} \mid a^{i \top} x \leq b_{i}, i \in I_{m}\right\},
$$

where for every $i \in I_{m}=\{1, \ldots, m\}$ the vector $a^{i}$ is a nonzero vector in $\mathbb{R}^{n}$ and $b_{i} \in \mathbb{R}$. Without loss of generality we assume that $P$ is full-dimensional, simple and there are no redundant constraints. For $I \subset I_{m}$, define

$$
F(I)=\left\{x \in P \mid a^{i \top} x=b_{i}, i \in I\right\}
$$

and

$$
C(I)=\left\{y \in \mathbb{R}^{n} \mid y=\sum_{i \in I} \mu_{i} a^{i}, \mu_{i} \geq 0, i \in I\right\} .
$$

Notice that $F(\emptyset)=P$ and $C(\emptyset)=\left\{0^{n}\right\}$. When $F(I) \neq \emptyset$, we call $F(I)$ a face of $P$. Let $\mathcal{I}$ be the collection of subsets $I$ of $I_{m}$ such that $F(I)$ is a face of $P$. For $x \in P$, define $I^{x}=\left\{i \in I_{m} \mid a^{i \top} x=b_{i}\right\}$, i.e. $F\left(I^{x}\right)$ is the (unique) face of $P$ of which $x$ is an interior point. Clearly, $C\left(I^{x}\right)$ is the normal cone of $P$ at $x \in P$, i.e. $C\left(I^{x}\right)=N(P, x)$. The next theorem gives a sufficient condition for the existence of a zero point of a mapping on $P$. 
Theorem 4.1 Let $P$ be a polytope and $\phi$ a mapping from $P$ to $\mathbb{R}^{n}$ satisfying the conditions stated before. Suppose that there exists a nonsingular $n \times n$ matrix $A$ and a collection of closed convex cones $Y(I), I \in \mathcal{I}$, such that $Y(I) \subset Y(J)$ whenever $J \subset I$ and satisfying the following two properties for every $x \in P$ :

4.1. The set $A \phi(x) \cap Y\left(I^{x}\right) \cap C\left(I^{x}\right)$ is either empty or contains $0^{n}$;

4.2. The set $A \phi(x) \cap Y\left(I^{x}\right) \neq \emptyset$.

Then there exists a zero point of $\phi$ in $P$.

Proof: Since $P$ is assumed to be simple and there are no redundant constraints, for every vector $v \in B^{n}$ there is a unique index set $I \in \mathcal{I}$ for which it holds that $v \in \operatorname{int}(C(I))$. For $v \in B^{n}$ define $\pi(v)=Y(I)$ for the unique $I \in \mathcal{I}$ for which $v \in \operatorname{int}(C(I))$. Clearly, $\pi(v)$ is a convex and closed set for every $v \in B^{n}$. To prove upper semi-continuity, let $\left(v^{k}, k \in \mathbb{N}\right)$, be a convergent sequence of points in $B^{n}$ and let $v$ be its limit point. For $k \in \mathbb{N}$, let $I_{k}$ be such that $v^{k} \in \operatorname{int}\left(C\left(I_{k}\right)\right)$, and let $I$ be such that $v \in \operatorname{int}(C(I))$. Since $I$ and all the $I_{k}$, $k \in \mathbb{N}$, are uniquely determined and $v^{k}$ converges to $v$, it holds that $I \subset I_{k}$ for sufficiently large $k \in \mathbb{N}$. Hence, $Y\left(I_{k}\right) \subset Y(I)$ for sufficiently large $k \in \mathbb{N}$ and therefore $\pi$ is an upper semi-continuous mapping. Moreover, because of Conditions 4.1 and 4.2, $\pi$ satisfies the Conditions 1 and 2 of Theorem 3.1. Consequently, there exists a zero point of $\phi$ on $P$.

The conditions in the theorem for a point $x$ in $P$ are completely determined by the set of indices that determines the face of $P$ in which $x$ lies.

\section{Intersection Theorems}

In this section we give a general intersection theorem on compact, convex sets. Let $X$ be again a non-empty, compact, convex set in $\mathbb{R}^{n}$. For some finite set of indices $\mathcal{J}$, let $\left\{D^{j} \mid j \in \mathcal{J}\right\}$ be a finite closed covering of $X$, i.e. for every $j \in \mathcal{J}$ the set $D^{j}$ is a closed, possibly empty, subset of $X$ and the union of all these sets is $X$. Let $\left\{c^{j} \mid j \in \mathcal{J}\right\}$ be some collection of vectors in $\mathbb{R}^{n}$. For a subset $J$ of $\mathcal{J}$, let $C(J)$ be defined by

$$
C(J)=\operatorname{con}\left\{c^{j} \mid j \in J\right\} .
$$

A collection $\left\{c^{j} \mid j \in J\right\}$ or the set $J$ itself is called balanced if $J$ is a non-empty subset of $\mathcal{J}$ and $0^{n} \in C(J)$. A point $x^{*} \in X$ is called an intersection point if $x^{*} \in \cap_{j \in J} D^{j}$ for some balanced set $J$. For $x \in X$ define the index set $J^{x}$ as

$$
J^{x}=\left\{j \in J \mid x \in D^{j}\right\}
$$


Because $\left\{D^{j} \mid j \in J\right\}$ is a covering of $X$, we have that $J^{x}$ is non-empty for every $x \in X$. By definition, $x^{*}$ is an intersection point if and only if the index set $J^{x^{*}}$ is balanced. The next theorem gives a sufficient condition for the existence of an intersection point.

Theorem 5.1 Let $\left\{D^{j} \mid j \in \mathcal{J}\right\}$ be a finite closed covering of a non-empty, compact, convex set $X$ in $\mathbb{R}^{n}$ and let $\left\{c^{j} \mid j \in \mathcal{J}\right\}$ be a collection of vectors in $\mathbb{R}^{n}$. Suppose that there exists a closed-valued, convex-valued, upper semi-continuous mapping $\pi: B^{n} \rightarrow \mathbb{R}^{n}$ such that for every $x \in X$ and $v \in N(X, x) \cap B^{n}$ the following two properties hold:

5.1. The set $C\left(J^{x}\right) \cap \pi(v) \cap\{y \mid y=\mu v, \mu \geq 0\}$ is either empty or contains $0^{n}$;

5.2. The set $C\left(J^{x}\right) \cap \pi(v) \neq \emptyset$.

Then there exists an intersection point.

Proof: Define $\phi: X \rightarrow \mathbb{R}^{n}$ by

$$
\phi(x)=C\left(J^{x}\right), x \in X .
$$

Since $\left\{D^{j} \mid j \in \mathcal{J}\right\}$ is a closed covering of $X$, we have that $\phi$ is an upper semi-continuous mapping. Moreover, for every $x \in X$, since $J^{x}$ is non-empty and $C\left(J^{x}\right)$ is the convex hull of a finite number of points, $\phi(x)$ is non-empty, convex, and compact. Because of Conditions 5.1 and 5.2 the mapping $\phi$ satisfies all the conditions of Theorem 3.1 and therefore there exists $x^{*} \in X$ satisfying $0^{n} \in \phi\left(x^{*}\right)$, i.e., $x^{*}$ is an intersection point.

In the remaining section we will show that several known intersection theorems like the ones of KKM (1929), Scarf (1967), Shapley (1973), and Ichiishi (1988) follow as special cases of Theorem 5.1.

The set $S^{n}=\left\{x \in \mathbb{R}_{+}^{n} \mid \sum_{i=1}^{n} x_{i}=1\right\}$ is called the unit simplex, which is a simple example of polytopes. For $h \in I_{n}, S_{h}^{n}$ denotes the facet $S_{h}^{n}=\left\{x \in S^{n} \mid x_{h}=0\right\}$, and for $T \subset I_{n}, S^{n}(T)=\cap_{h \in T} S_{h}^{n}$. We define the $j$-th unit vector in $\mathbb{R}^{n}$ by $e^{j}$. The first result is the classical KKM-lemma.

Example 5.2 Let $\left\{D^{j} \mid j \in I_{n}\right\}$ be a collection of closed sets covering the unit simplex $S^{n}$ satisfying that for every $T \subset I_{n}$, the face $S^{n}(T)$ is contained in $\cup_{j \notin T} D^{j}$. Then $\cap_{j \in I_{n}} D^{j} \neq$ $\emptyset$.

Proof: From the definition of the simplex, $S^{n}=\left\{x \in \mathbb{R}^{n} \mid-x_{i} \leq 0, i \in I_{n}\right.$, and $\left.1^{n} \cdot x=1\right\}$, it follows immediately that

$$
N(X, x)=\left\{v \in \mathbb{R}^{n} \mid v=\alpha 1^{n}-\sum_{\left\{i \mid x_{i}=0\right\}} \beta_{i} e^{i}, \alpha \in \mathbb{R}, \beta_{i} \geq 0\right\} .
$$


We define $\pi(v)=\left\{y \in \mathbb{R}^{n} \mid y^{\top} v \leq 0\right\}$ and $c^{j}=\frac{1}{n} 1^{n}-e^{j}, j \in I_{n}$. Notice that the collection $\left\{c^{j} \mid j \in J\right\}$ is balanced if and only if $J=I_{n}$. To show the KKM-lemma, it remains to verify the two conditions of Theorem 5.1 .

Since $\pi(v) \cap\{y \mid y=\mu v, \mu \geq 0\}$ equals $0^{n}$, Condition 1 is clearly satisfied.

Consider $x \in S^{n}$. If $x \in \operatorname{int}\left(S^{n}\right)$, then $N(X, x)=\left\{v \in \mathbb{R}^{n} \mid v=\alpha 1^{n}, \alpha \in \mathbb{R}\right\}$, so $v \in N(X, x) \backslash\left\{0^{n}\right\}$ implies $\pi(v)=\left\{x \in \mathbb{R}^{n} \mid 1^{n} \cdot x=0\right\}$, so $C\left(J^{x}\right) \cap \pi(v)=C\left(J^{x}\right) \neq \emptyset$. If $x \in \operatorname{bd}\left(S^{n}\right)$, say $x \in S^{n}(T)$ with $T=\left\{j \mid x_{j}=0\right\}$, then $x \in D^{j}$ for some $j \in I_{n} \backslash T$. The corresponding $c^{j} \in C\left(J^{x}\right)$ satisfies $c_{j}^{j}=\frac{1}{n}-1$ and $c_{k}^{j}=\frac{1}{n}$ if $k \neq j$. Whenever $v \in N(X, x)$ it holds that $v=\alpha 1^{n}-\sum_{i \in T} \beta_{i} e^{i}$, so $v^{\top} c^{j}=-\sum_{i \in T} \beta_{i} \frac{1}{n} \leq 0$. It follows that $c^{j} \in \pi(v)$.

The next example is due to Scarf (1967) and can be viewed as a dual version of the KKM lemma.

Example 5.3 Let $\left\{D^{j} \mid j \in I_{n}\right\}$ be a collection of closed sets covering the unit simplex $S^{n}$ satisfying that for every $j \in I_{n}$, the facet $S_{j}^{n}$ is contained in $D^{j}$. Then $\cap_{j \in I_{n}} D^{j} \neq \emptyset$.

Proof: We define $\pi(v)=\left\{y \in \mathbb{R}^{n} \mid y^{\top} v \leq 0\right\}$ and $c^{j}=e^{j}-\frac{1}{n} 1^{n}, j \in I_{n}$. Notice that the collection $\left\{c^{j} \mid j \in J\right\}$ is balanced if and only if $J=I_{n}$. To show the Scarf-lemma, it remains to verify the two conditions of Theorem 5.1 .

Condition 1 is satisfied for the same reason as in Example 5.2.

Consider $x \in S^{n}$. If $x \in \operatorname{int}\left(S^{n}\right)$, then $v \in N(X, x) \backslash\left\{0^{n}\right\}$ implies $\pi(v)=\{x \in$ $\left.\mathbb{R}^{n} \mid 1^{n} \cdot x=0\right\}$, so $C\left(J^{x}\right) \cap \pi(v)=C\left(J^{x}\right) \neq \emptyset$. If $x \in \operatorname{bd}\left(S^{n}\right)$, say $x \in S^{n}(T)$ with $T=\left\{j \mid x_{j}=0\right\}$, then $x \in D^{j}$ for all $j \in T$. The vector $\bar{c} \in C\left(J^{x}\right)$ defined by $\bar{c}=\sum_{j \in T} \frac{1}{|T|} c^{j}$ satisfies $\bar{c}_{j}=\frac{1}{|T|}-\frac{1}{n}$ if $j \in T$ and $\bar{c}_{j}=-\frac{1}{n}$ if $j \in I_{n} \backslash T$. Whenever $v \in N(X, x)$ it holds that $v=\alpha 1^{n}-\sum_{j \in T} \beta_{j} e^{j}$, so $v^{\top} \bar{c}=-\sum_{j \in T}\left(\frac{1}{|T|}-\frac{1}{n}\right) \beta_{j} \leq 0$. It follows that $\bar{c} \in \pi(v)$.

We continue with the Shapley lemma. We define the collection of non-empty subsets of $I_{n}$ by $\mathcal{I}_{n}$. For $S \subset \mathcal{I}_{n}$, we define $e^{S} \in \mathbb{R}^{n}$ as the vector satisfying $e_{i}^{S}=1$ if $i \in S$ and $e_{i}^{S}=0$ otherwise. We say that a collection $\mathcal{B}=\left\{B_{1}, \ldots, B_{k}\right\}$ of members of $\mathcal{I}$ is set-balanced if there exist non-negative numbers $\lambda_{j}, j=1, \ldots, k$, such that $\sum_{j=1}^{k} \lambda_{j} e^{B_{j}}=1^{n}$.

Example 5.4 Let $\left\{D^{S} \mid S \in \mathcal{I}_{n}\right\}$ be a collection of closed sets covering the unit simplex $S^{n}$ satisfying that for every $T \subset I_{n}$, the face $S^{n}(T)$ is contained in $\cup_{S \subset I_{n} \backslash T} D^{S}$. Then there is a set-balanced family $\mathcal{B}=\left\{B_{1}, \ldots, B_{k}\right\}$ of elements of $\mathcal{I}_{n}$ for which $\cap_{j=1}^{k} D^{B_{j}} \neq \emptyset$.

Proof: We define $\pi(v)=\left\{y \in \mathbb{R}^{n} \mid y^{\top} v \leq 0\right\}$ and $c^{S}=\frac{1}{n} 1^{n}-e^{S}, S \in \mathcal{I}_{n}$. Notice that the collection $\left\{c^{S_{1}}, \ldots, c^{S_{k}}\right\}$ is balanced if and only if $\left\{S_{1}, \ldots, S_{k}\right\}$ is set-balanced. To show the KKMS-lemma, it remains to verify the two conditions of Theorem 5.1.

Since $\pi(v) \cap\{y \mid y=\mu v, \mu \geq 0\}$ equals $0^{n}$, Condition 1 is clearly satisfied. 
Consider $x \in S^{n}$. If $x \in \operatorname{int}\left(S^{n}\right)$, then $v \in N(X, x) \backslash\left\{0^{n}\right\}$ implies $\pi(v)=\{x \in$ $\left.\mathbb{R}^{n} \mid 1^{n} \cdot x=0\right\}$, so $C\left(J^{x}\right) \cap \pi(v)=C\left(J^{x}\right) \neq \emptyset$. If $x \in \operatorname{bd}\left(S^{n}\right)$, say $x \in S^{n}(T)$ with $T=\left\{j \mid x_{j}=0\right\}$, then $x \in D^{S}$ for some $S \subset I_{n} \backslash T$. The corresponding $c^{S} \in C\left(J^{x}\right)$ satisfies $c_{j}^{S}=\frac{1}{n}-\frac{1}{|S|}$ if $j \in S$ and $c_{j}^{S}=\frac{1}{n}$ if $j \notin S$. Whenever $v \in N(X, x)$ it holds that $v=\alpha 1^{n}-\sum_{i \in T} \beta_{i} e^{i}$, so $v^{\top} c^{S}=-\sum_{i \in T} \beta_{i} c_{i}^{S}=-\sum_{i \in T} \beta_{i} \frac{1}{n} \leq 0$. It follows that $c^{S} \in \pi(v)$.

The next result is due to Ichiishi (1988) which can be seen as a dual version of Shapley's intersection lemma.

Example 5.5 Let $\left\{D^{S} \mid S \in \mathcal{I}_{n}\right\}$ be a collection of closed sets covering the unit simplex $S^{n}$ satisfying that for every $T \in \mathcal{I}_{n}$, the face $S^{n}(T)$ is contained in $\cup_{T \subset S} D^{S}$. Then there is a set-balanced family $\mathcal{B}=\left\{B_{1}, \ldots, B_{k}\right\}$ of elements of $\mathcal{I}_{n}$ for which $\cap_{j=1}^{k} D^{B_{j}} \neq \emptyset$.

Proof: We define $\pi(v)=\left\{y \in \mathbb{R}^{n} \mid y^{\top} v \leq 0\right\}$ and $c^{S}=e^{S}-\frac{1}{n} 1^{n}, S \in \mathcal{I}_{n}$. Notice that the collection $\left\{c^{S_{1}}, \ldots, c^{S_{k}}\right\}$ is balanced if and only if $\left\{S_{1}, \ldots, S_{k}\right\}$ is set-balanced. To show the Ichiishi-lemma, it remains to verify the two conditions of Theorem 5.1.

Condition 1 is satisfied for the same reason as in Example 5.2.

Consider $x \in S^{n}$. If $x \in \operatorname{int}\left(S^{n}\right)$, then $v \in N(X, x) \backslash\left\{0^{n}\right\}$ implies $\pi(v)=\{x \in$ $\left.\mathbb{R}^{n} \mid 1^{n} \cdot x=0\right\}$, so $C\left(J^{x}\right) \cap \pi(v)=C\left(J^{x}\right) \neq \emptyset$. If $x \in \operatorname{bd}\left(S^{n}\right)$, say $x \in S^{n}(T)$ with $T=\left\{j \mid x_{j}=0\right\}$, then $x \in D^{S}$ for some $S \supset T$. The vector $c^{S} \in C\left(J^{x}\right)$ satisfies $c_{j}^{S}=\frac{1}{|S|}-\frac{1}{n}$ if $j \in S$ and $c_{j}^{S}=-\frac{1}{n}$ if $j \in I_{n} \backslash S$. Whenever $v \in N(X, x)$ it holds that $v=\alpha 1^{n}-\sum_{j \in T} \beta_{j} e^{j}$, so $v^{\top} c^{S}=-\sum_{j \in T}\left(\frac{1}{|S|}-\frac{1}{n}\right) \beta_{j} \leq 0$. It follows that $c^{S} \in \pi(v)$.

Finally, we will show that a quite general intersection theorem of van der Laan, Talman and Yang (1999) also follows from Theorem 5.1 as a particular case. To state their result, we first define, for $I \subset I_{m}$, the set $A^{*}(I)$ by

$$
A^{*}(I)=\left\{y \in \mathbb{R}^{n} \mid y^{\top} x \leq 0 \text { for all } x \in A(I)\right\} .
$$

Their theorem reads as follows.

Theorem 5.6 Let $\left\{D^{j} \mid j \in \mathcal{J}\right\}$ be a finite closed covering of a full-dimensional polytope $P=\left\{x \in \mathbb{R}^{n} \mid a^{i^{\top}} x \leq \alpha_{i}, i \in I\right\}$ and let $\left\{c^{j} \mid j \in \mathcal{J}\right\}$ be a collection of vectors in $\mathbb{R}^{n}$. Suppose that for every $x \in b d(P)$ it holds that $C\left(J^{x}\right) \cap A^{*}\left(I^{x}\right) \neq \emptyset$. Then there exists a balanced set $J \subset \mathcal{J}$ for which $\cap_{j \in J} D^{j} \neq \emptyset$.

Proof: Define $\pi(v)=\left\{y \in \mathbb{R}^{n} \mid y^{\top} v \leq 0\right\}$.

Since $P$ is a full-dimensional polytope, it follows that for $x \in \operatorname{int}(P)$ it holds that $N(X, x)=\left\{0^{n}\right\}$, so Conditions 1 and 2 of Theorem 5.1 are obviously satisfied. 
Consider $x \in \mathrm{bd}(P)$. Condition 1 of Theorem 5.1 is satisfied for the same reason as in Example 5.2. Let $y$ be an element of $C\left(J^{x}\right) \cap A^{*}\left(I^{x}\right)$. Then $y^{\top} v \leq 0$ for all $v \in N(X, x)$, so $y \in C\left(J^{x}\right) \cap \pi(v)$ for all $v \in N(X, x)$, and Condition 2 of Theorem 5.1 is satisfied as well. It follows that there is an intersection point, i.e. there exists a balanced set $J \subset \mathcal{J}$ for which $\cap_{j \in J} D^{j} \neq \emptyset$.

Theorem 5.1 generalizes Theorem 5.6 in two respects. First, it treats the case of an arbitrary non-empty, compact, and convex set $X$, and thereby generalizes the assumption that $X$ be a polytope. Secondly, it weakens the boundary condition.

Theorem 5.6 contains generalizations of the lemmas of KKM, Scarf, Shapley and Ichiishi to the polytope as special cases, as well as lemmas on the cube by Freund (1986) and lemmas on the polytope by Ichiishi and Idzik (1991). Since Theorem 5.6 is a special case of Theorem 5.1, these results follow as special cases of Theorem 5.1 as well. We also refer to Gale (1984), Herings and Talman (1998) and Yang (1999, 2001) for other types of intersection results.

\section{References}

[1] L.E.J. Brouwer, "Über Abbildung von Mannigfaltigkeiten”, Mathematische Annalen 71 (1912) 97-115.

[2] F.E. Browder, "On continuity of fixed points under deformation of continuous mapping", Summa Brasiliensis Mathematicae 4 (1960) 183-191.

[3] B.C. Eaves, "On the basic theory of complementarity", Mathematical Programming 1 (1971) 68-75.

[4] B.C. Eaves, "Homotopies for computation of fixed points", Mathematical Programming 3 (1972) 1-22.

[5] R.W. Freund, "Combinatorial Theorems on the Simplotope that Generalize Results on the Simplex and Cube," Mathematics of Operations Research 11 (1986) 169-179.

[6] Gale, D, "Equilibrium in a discrete exchange economy with money," International Journal of Game Theory 13 (1984) 61-64.

[7] P.J.J. Herings and A.J.J. Talman, "Intersection theorems with a continuum of intersection points", Journal of Optimization Theory and Applications 96 (1998) 311-335.

[8] P.J.J. Herings, G. van der Laan and A.J.J. Talman, Quantity constrained equilibria, METEOR Research Memorandum 01/23, Universiteit Maastricht (2001) 1-27. 
[9] P.J.J. Herings, A.J.J. Talman and Z. Yang, "The computation of a continuum of constrained equilibria", Mathematics of Operations Research 21 (1996) 675-696.

[10] P.J.J. Herings, A.J.J. Talman and Z. Yang, "Variational inequality problems with a continuum of solutions: existence and computation", SIAM Journal on Control and Optimization 39 (2001) 1852-1873.

[11] T. Ichiishi, "Alternative Version of Shapley's Theorem on Closed Coverings of a Simplex," Proceedings of the American Mathematical Society 104 (1988) 759-763.

[12] T. Ichiishi and A. Idzik, "Closed Covers of Compact Convex Polyhedra," International Journal of Game Theory 20 (1991) 161-169.

[13] S. Kakutani, "A generalization of Brouwer's fixed point theorem", Duke Mathematical Journal 8 (1941) 457-459.

[14] B. Knaster, C. Kuratowski, and C. Mazurkiewicz, "Ein Beweis des Fixpunktsatzes für n-dimensionale Simplexe", Fundamenta Mathematicae 14 (1929) 132-137.

[15] G. van der Laan, A.J.J. Talman, and Z. Yang, "Intersection theorems on polytopes", Mathematical Programming 84 (1999) 25-38.

[16] H. Scarf, "The approximation of fixed points of a continuous mapping", SIAM Journal on Applied Mathematics 15 (1967) 1328-1343.

[17] L.S. Shapley, "On Balanced Games without Side Payments," in T.C. Hu and S.M. Robinson (eds.), Mathematical Programming, Academic Press, New York, USA (1973), pp. 261-290.

[18] Z. Yang, Computing Equilibria and Fixed Points, Kluwer Academic Publishers, Boston, 1999.

[19] Z. Yang, "An intersection theorem on an unbounded set and its application to the fair allocation problem", Journal of Optimization Theory and Applications 110 (2001) 429-443. 